\title{
Editorial: DNA Replication Origins in Microbial Genomes
}

\author{
Feng Gao 1, 2, 3* \\ ${ }^{1}$ Department of Physics, Tianjin University, Tianjin, China, ${ }^{2}$ Key Laboratory of Systems Bioengineering, Ministry of Education, \\ Tianjin University, Tianjin, China, ${ }^{3}$ SynBio Research Platform, Collaborative Innovation Center of Chemical Science and \\ Engineering, Tianjin, China
}

Keywords: archaea, bacteria, yeast, replication origin, DNA replication, replication regulation, orisome, regulatory proteins

\section{The Editorial on the Research Topic}

\section{DNA Replication Origins in Microbial Genomes}

In all three domains of life, DNA replication initiates on defined genome sites, termed replication origins. In bacteria, replication typically initiates from a single replication origin (oriC). In eukaryotic genomes, replication initiates from significantly more replication origins, ranging from hundreds in yeast to tens of thousands in human (Gao et al., 2012). Within the archaeal domain, multiple replication origins have been identified in Sulfolobus species, haloarchaea etc. (Lundgren et al., 2004; Robinson et al., 2004; Wu et al.; Yang et al., 2015). The research on replication origins is important not only in providing insights into the structure and function of the replication origins but also in understanding the regulatory mechanisms of the initiation step in DNA replication. Therefore, intensive studies, by in silico analyses as well as in vivo and in vitro experiments, have been carried out in the last two decades.

Based on the sequence-derived features, various in silico approaches have been developed to identify microbial replication origins (Frank and Lobry, 2000; Breier et al., 2004; Mackiewicz et al., 2004; Zhang and Zhang, 2005; Worning et al., 2006; Gao and Zhang, 2007, 2008a; Gao et al., 2013; Gao, 2014). For example, the locations of replication origins sites have been predicted for thousands of bacterial genomes by Ori-Finder, a web-based system for finding oriCs in bacterial genomes (Gao and Zhang, 2007; Gao et al., 2013). A new version of Ori-Finder for archaea, Ori-Finder 2, has been developed to predict oriCs in archaeal genomes automatically (Luo et al.). To confirm the predicted replication origins, it is important to choose a most suitable experimental strategy. Song et al. summarize the main existing experimental methods to determine the replication origin regions and their practical applications (Song et al.). As a study from in silico to in vitro, the experimental supports are provided for the identified replication origins in Cyanothece ATCC 51142 (Gao and Zhang, 2008b), and their interactions with the initiator protein DnaA (Huang et al.).

In spite of a great variety of origin sequences across species, all bacterial replication origins contain the information necessary to guide assembly of the DnaA protein complex at oriC, triggering the unwinding of DNA and the beginning of replication. Therefore, oriC-encoded instructions should be interpreted particularly in the context of replication initiation and its regulation (Wolanski et al.). Wolanski et al. show that oriC-encoded instructions allow not only for initiation but also for precise regulation of replication initiation and coordination of chromosomal replication with the cell cycle (also in response to environmental signals; Wolanski et al.). FrimodtMoller et al. find control regions for chromosome replication are conserved with respect to sequence and location among Escherichia coli strains (Frimodt-Moller et al.). Based on the single origin usage strategy that distinguishes bacteria, Marczynski et al. redefine the bacterial origins 
as centralized information processors, and describe how negative-feedback, phospho-relay, and chromosomepartitioning systems act to regulate chromosome replication (Marczynski et al.). On the other hand, the in silico analyses show that some bacteria, although very few, may have multiple origins of replication per chromosome (Frank et al., 2015; Gao), and the recent work also suggests that there are multiple replication origins in Synechocystis that fire asynchronously, as in eukaryotic nuclear chromosomal replication (Ohbayashi et al., 2015).

For eukaryotic organisms, replication origins are best characterized in the unicellular eukaryote budding yeast Saccharomyces cerevisiae and the fission yeast Schizosaccharomyces pombe. With the recent development of genome-wide approaches, the number of yeast species involved in ORIs research has increased dramatically, which has created opportunities for the sequence, protein, and comparative analysis of replication origins in yeast genomes (Li et al.; Zheng et al.; Peng et al.).

The Frontiers in Microbiology Research Topic on DNA replication origins in microbial genomes is devoted to address the issues mentioned above, and aims to provide

\section{REFERENCES}

Breier, A. M., Chatterji, S., and Cozzarelli, N. R. (2004). Prediction of Saccharomyces cerevisiae replication origins. Genome Biol. 5:R22. doi: 10.1186/gb-2004-5-4-r22

Frank, A. C., and Lobry, J. R. (2000). Oriloc: prediction of replication boundaries in unannotated bacterial chromosomes. Bioinformatics 16, 560-561. doi: 10.1093/bioinformatics/16.6.560

Frank, O., Göker, M., Pradella, S., and Petersen, J. (2015). Ocean's Twelve: flagellar and biofilm chromids in the multipartite genome of Marinovum algicola DG898 exemplify functional compartmentalization. Environ. Microbiol. 17, 4019-4034. doi: 10.1111/1462-2920.12947

Gao, F. (2014). Recent advances in the identification of replication origins based on the Z-curve method. Curr. Genomics 15, 104-112. doi: $10.2174 / 1389202915999140328162938$

Gao, F., Luo, H., and Zhang, C. T. (2012). DeOri: a database of eukaryotic DNA replication origins. Bioinformatics 28, 1551-1552. doi: 10.1093/bioinformatics/bts151

Gao, F., Luo, H., and Zhang, C. T. (2013). DoriC 5.0: an updated database of oriC regions in both bacterial and archaeal genomes. Nucleic Acids Res. 41, D90-D93. doi: 10.1093/nar/gks990

Gao, F., and Zhang, C. T. (2007). DoriC: a database of oriC regions in bacterial genomes. Bioinformatics 23, 1866-1867. doi: 10.1093/bioinformatics/btm 255

Gao, F., and Zhang, C. T. (2008a). Ori-Finder: a web-based system for finding oriCs in unannotated bacterial genomes. BMC Bioinformatics 9:79. doi: 10.1186/1471-2105-9-79

Gao, F., and Zhang, C. T. (2008b). Origins of replication in Cyanothece 51142. Proc. Natl. Acad. Sci. U.S.A. 105, E125; author reply E126-127. doi: 10.1073/pnas.0809987106

Lundgren, M., Andersson, A., Chen, L., Nilsson, P., and Bernander, R. (2004). Three replication origins in Sulfolobus species: synchronous initiation of chromosome replication and asynchronous termination. Proc. Natl. Acad. Sci. U.S.A. 101, 7046-7051. doi: 10.1073/pnas.0400656101 a comprehensive overview of the current research in this field.

\section{DEDICATION}

This article is dedicated to the 120th Anniversary of Tianjin University (formerly Peiyang University), the first modern higher education university in China.

\section{ACKNOWLEDGMENTS}

The author would like to thank Prof. Chun-Ting Zhang for the invaluable assistance and inspiring discussions, the international editors and reviewers from over 10 countries for their excellent assistance and constructive comments on the manuscripts, and the Frontiers in Microbiology team for their continued support and assistance. The present work was supported in part by National Natural Science Foundation of China (Grant Nos. 31571358, 31171238, 30800642, and 10747150), Program for New Century Excellent Talents in University (No. NCET12-0396), and the China National 863 High-Tech Program (2015AA020101).

Mackiewicz, P., Zakrzewska-Czerwinska, J., Zawilak, A., Dudek, M. R., and Cebrat, S. (2004). Where does bacterial replication start? Rules for predicting the oriC region. Nucleic Acids Res. 32, 3781-3791. doi: 10.1093/nar/ gkh699

Ohbayashi, R., Watanabe, S., Ehira, S., Kanesaki, Y., Chibazakura, T., and Yoshikawa, H. (2015). Diversification of DnaA dependency for DNA replication in cyanobacterial evolution. ISME J. doi: 10.1038/ismej.2015.194. [Epub ahead of print].

Robinson, N. P., Dionne, I., Lundgren, M., Marsh, V. L., Bernander, R., and Bell, S. D. (2004). Identification of two origins of replication in the single chromosome of the archaeon Sulfolobus solfataricus. Cell 116, 25-38. doi: 10.1016/S0092-8674(03)01034-1

Worning, P., Jensen, L. J., Hallin, P. F., Staerfeldt, H. H., and Ussery, D. W. (2006) Origin of replication in circular prokaryotic chromosomes. Environ. Microbiol. 8, 353-361. doi: 10.1111/j.1462-2920.2005.00917.x

Yang, H., Wu, Z., Liu, J., Liu, X., Wang, L., Cai, S., et al. (2015). Activation of a dormant replication origin is essential for Haloferax mediterranei lacking the primary origins. Nat. Commun. 6, 8321. doi: 10.1038/ncomm s9321

Zhang, R., and Zhang, C. T. (2005). Identification of replication origins in archaeal genomes based on the Z-curve method. Archaea 1, 335-346. doi: $10.1155 / 2005 / 509646$

Conflict of Interest Statement: The author declares that the research was conducted in the absence of any commercial or financial relationships that could be construed as a potential conflict of interest.

Copyright (c) 2016 Gao. This is an open-access article distributed under the terms of the Creative Commons Attribution License (CC BY). The use, distribution or reproduction in other forums is permitted, provided the original author(s) or licensor are credited and that the original publication in this journal is cited, in accordance with accepted academic practice. No use, distribution or reproduction is permitted which does not comply with these terms. 